\title{
Association between Q115L of PINK1 Gene Polymorphism and Parkinson's Disease: A Meta-Analysis
}

\author{
Zhicheng Liu¹, Zhiguo Liu²
}

\author{
${ }^{1}$ Medical College of Qingdao University, Qingdao, China \\ ${ }^{2}$ Department of Bone Joint and Sports Medicine,Qingdao Municipal Hospital, Shandong Province, China
}

\begin{abstract}
Many case-control studies have researched the associations between PINK1 Q115L polymorphisms and the risk of Parkinson's disease (PD), but the results exist controversies. We performed a meta-analysis to assess the possible association between the PINK1 Q115L gene polymorphisms and PD. Here we searched the Pubmed, CNKI, CBM and Web of Science databases up to 2015 to identify published related studies. The meta-analysis was then conducted to analysis the possible associations between the PINK1 Q115L polymorphisms and PD. A total of six studies were included in the meta-analysis. The crude odds ratios (ORs) with 95\% confidence intervals (95\% CI) were calculated to evaluate the association. Heterogeneity among studies was evaluated using the $\mathrm{I}^{2}$ and Egger's test was used to evaluate publication bias. Sensitivity analysis was also performed. After exclusion of articles deviating from HWE in controls. The meta-analysis also showed no significant association between the $\mathrm{T}$ allele and increased risk of PD in allele model (FEM: OR=0.790, 95\% CI=0.568-1.098); dominant model (FEM: OR=1.344, 95\% $\mathrm{CI}=0.952-1.897$ ); heterozygote model (FEM: $\mathrm{OR}=0.736,95 \% \mathrm{CI}=0.519-1.043$ ). In subgroup analysis, for Caucasian, there was no significantly association in all three models. Our suggested that the PINK1 Q115L polymorphism might not be associated with PD.
\end{abstract}

Keywords: PINK1, Q115L, Parkinson's Disease, Meta-Analysis, Mitochondria

\section{Introduction}

Parkinson's disease (PD), as the second most prevalent neurodegenerative disease, affects as many as $1-2 \%$ of the worldwide population over 60[1-3]. The cardinal features of PD are tremor, rigidity, bradykinesia, and postural instability.

It is characterized by selective loss of the dopaminergic neurons in the substantia nigra pars compacta $\left(\mathrm{SN}_{\mathrm{pc}}\right)$, a profound loss of dopamine (DA) in the striatum, and the presence of intracytoplasmic inclusions called Lewy bodies (LB) in the remaining dopaminergic neurons[4-5].

Although the majority of PD cases are sporadic, a small number $(<10 \%)$ display familial clustering[6]. Discovering PD genetic risk factors is important for understanding its etiology. Mutations in five genes have been identified to influence PD risk in fewer than $5 \%$ of those with PD[7]. Three, PARK2 (parkin), PARK7 (DJ1), and PINK1, are typically transmitted with autosomal recessive inheritance and two, SNCA and $L R R K 2$, are inherited in an autosomal dominant fashion. Mutations in coding regions of PINK1 gene are of ten implicated as the most common cause of early-onset Parkinson's disease.

PINK1 is synthesized as a 581 amino acid protein containing a mitochondrial localization sequence, a predicted transmembrane (TM) domain and a serine/threonine kinase domain[8]. Mutations within the kinase domain and $\mathrm{C}$-terminus of the protein reduce PINK1 kinase activity, and this loss of kinase function is thought to be responsible for PD pathogenesis[9]. However, a series of PD-associated mutations lie within the N-terminal region of the PINK1 protein and it is mechanistically unclear how these mutations would accountfor loss of kinase function[10-12]. A study had showed that the PINK1 PD mutations C92F, Q115L and R147H, located close to the cleavage site, cause an accumulation of the FL-PINK1 protein, which results in an intermediate mitochondrial phenotype[11, 13-17]. Although more attentions have been paid to the Q115L variants in PINK1 and their associations with 
$\mathrm{PD}$, the results in the publication remain controversial, because the sample capacity is sparse. Hence, we performed a meta-analysis of the studies that presented original data to examine whether the PINK1 Q115L polymorpphisms have association with PD.

\section{Materials and methods}

\section{Literature search}

A search was conducted for relevant available articles published in English or Chinese from four databases: (1) PubMed (1990-2015); (2) China National Knowledge Infrastructure (CNKI) (1990-2015); (3) China Biology Medical literature database (CBM) (1990-2015); and (4) Web of Science (ISI) (1990-2015). The following terms and keywords were used:"Parkinson's disease" (or "PD"), "PINK1" (or "PTEN induced putative kinase 1"), and "polymorphism" (or "variant"). Additional studies not captured by our database searches were identified by reviewing the bibliographies of relevant articles as well as those of relevant studies.

\section{Selection criteria}

The inclusion criteria were as follows: (1) full-text articles; (2) case-control study that focused on the relationships of PINK1 Q115L variants with PD; (3) provided sufficient data about PINK1 Q115L genotypes and genotype distributions to estimate the odds ratio (OR) with $95 \%$ confidence intervals $(95 \%$ $\mathrm{CI})$; Studies were excluded for the following reasons: (1) irrelevant papers; (2) not case-control studies; (3) based on incomplete data; (4) letters, reviews, meta-analyses.

\section{Statistical analysis}

Departure from Hardy-Weinberg equilibrium (HWE) for the Q115L genotype distributions of PINK gene in controls was tested by $\chi^{2}$ analysis with exact probability, and $p<0.05$ was considered as departure from HWE. The risk of PD associated with Q115L polymorphism was estimated for each study by ORs with $95 \%$ CI. We estimated the association under three different types of ORs, including Q115L allele model (A vs.T), dominant model (AA vs. TT+TA) and heterozygote model (AA vs. TA). $\mathrm{I}^{2}$-statistics was used to assess between-study heterogeneity. If abundant heterogeneity is present $\left(\mathrm{I}^{2}>50 \%\right)$ [18], the random effect model (REM) was used as the pooling method; otherwise, the fixed effect model (FEM) was used. Publication bias was estimated using modified Egger's linear regression test proposed by Peters et al[19-20]. The leave-one-out sensitive analysis [21] was carried out to evaluate the key studies with substantial impact on between-study heterogeneity. An analysis of influence was conducted to describe how robust the pooled estimator is to removal of individual studies. If the main estimate of an individual study's omitted analysis lies outside the $95 \%$ CI of the combined analysis, it is suspected of excessive influence. Publication bias was estimated

using the Begg's test. All statistical analyses were performed by using STATA version 12.0 (Stata Corporation, College Station, Texas 77845 USA).

\section{Result}

\section{Characteristics of eligible studies}

A total of 6 articles that studied the association between PINK1 Q115L polymorphism and Parkinson's disease were identified including 1,057 cases and 1,529 controls(Fig 1). The main characteristics of the eligible studies are described in Table 1. 
Association between Q115L of PINK1 Gene Polymorphism and Parkinson's Disease: A Meta-Analysis

\begin{tabular}{|c|c|c|c|c|c|c|c|c|c|c|c|c|}
\hline \multirow[t]{2}{*}{ Author } & \multirow[t]{2}{*}{ Year } & \multirow[t]{2}{*}{ Country } & \multirow[t]{2}{*}{ Ethnicity } & \multicolumn{2}{|c|}{ Sample size } & \multicolumn{3}{|c|}{ Case } & \multicolumn{3}{|c|}{ Control } & \multirow{2}{*}{$\begin{array}{l}P \text { for } \\
\text { HWE }\end{array}$} \\
\hline & & & & Case & Control & $\pi$ & TA & $\mathrm{AA}$ & $\pi$ & $\mathrm{TA}$ & AA & \\
\hline Bonifati & 2005 & mixed & $\longrightarrow$ & 103 & 90 & 0 & 9 & 94 & 1 & 12 & 77 & 0.5 \\
\hline Klein & 2005 & Italy & Caucasian & 65 & 100 & 0 & 3 & 62 & 0 & 6 & 94 & 0.75 \\
\hline Schlitter & 2006 & Norway & Caucasian & 90 & 136 & 3 & 7 & 80 & 0 & 18 & 118 & 0.4 \\
\hline Schlitter & 2007 & Germany & Caucasian & 85 & 210 & 0 & 6 & 79 & 4 & 16 & 190 & $<0.01$ \\
\hline Toft & 2007 & Norway & Caucasian & 133 & 350 & 0 & 16 & 117 & 0 & 39 & 311 & 0.26 \\
\hline Ishihara-Paul & 2008 & Tunisia & Africa & 331 & 367 & 0 & 3 & 328 & 1 & 13 & 353 & 0.02 \\
\hline Brooks & 2008 & USA & Caucasian & 250 & 276 & 0 & 17 & 233 & 0 & 12 & 244 & 0.3 \\
\hline
\end{tabular}

Table 1 Characteristics of PINK1 gene Q115L polymorphism genotype distributions in studies included in the meta-analysis. $P$ for Hardy-Weinberg equilibrium (HWE) in control group

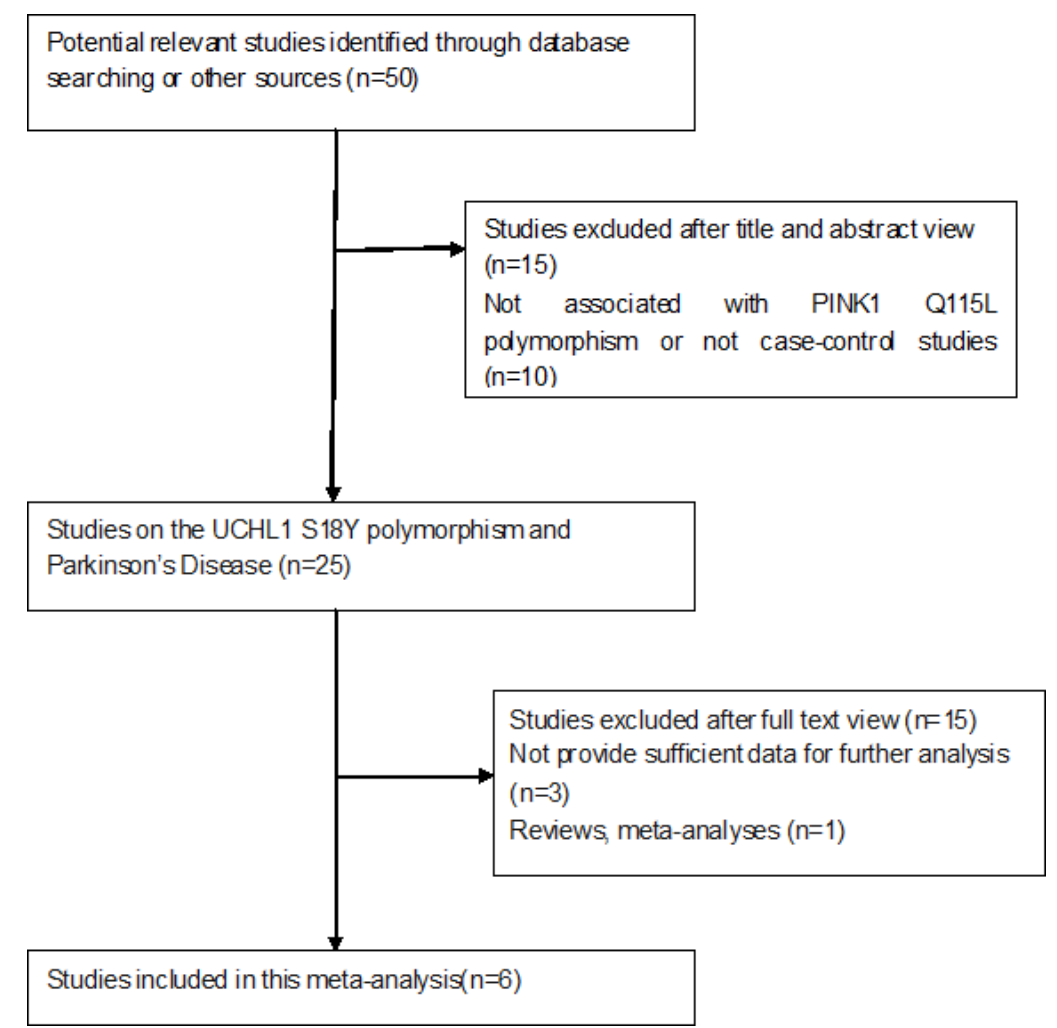

Fig. 1 Flow chart of publication selection procedure

\section{Quantitative Synthesis}

The results of the pooled analysis are summarized in Table 2. The meta-analysis also showed a significant association between the $\mathrm{T}$ allele and increased risk of $\mathrm{PD}$ in allele model (FEM: OR=0.693, 95\% $\mathrm{CI}=0.515-0.933$ ); dominant model (FEM: $\mathrm{OR}=1.482$, 95\% CI=1.085-2.023); heterozygote model (FEM:
$\mathrm{OR}=1.491,95 \% \mathrm{CI}=1.085-2.048)$. In subgroup analysis, for Caucasian, the $\mathrm{T}$ allele was not found contributing to significantly to increased PD risk in allele model (FEM: OR=0.803, 95\% CI=0.577-1.117); dominant model (FEM: OR=1.297, 95\% CI=0.917-1.835); heterozygote model (FEM: OR=1.333, 95\% $\mathrm{CI}=0.935-1.899)$. After exclusion of articles deviating 
Association between Q115L of PINK1 Gene Polymorphism and Parkinson's Disease: A Meta-Analysis

from HWE in controls[14, 16], the associations in the above-mentioned inherited models were not significant in allele model (FEM: OR $=0.790$, 95\% $\mathrm{CI}=0.568-1.098)$; dominant model (FEM: OR=1.344,
95\% CI=0.952-1.897); heterozygote model (FEM: $\mathrm{OR}=0.736,95 \% \mathrm{CI}=0.519-1.043)$. In subgroup analysis, for Caucasian, there was no significantly association in all three models(Fig 2).

\begin{tabular}{|c|c|c|c|c|c|c|c|c|}
\hline \multirow[t]{2}{*}{ Data } & \multirow[t]{2}{*}{ SNPs } & \multirow[t]{2}{*}{ Contrast } & \multicolumn{3}{|c|}{ Odds ratio } & \multirow[t]{2}{*}{ Model } & \multicolumn{2}{|c|}{ Heterogenity } \\
\hline & & & $\overline{\mathrm{OR}}$ & (95CI) & $\mathrm{P}_{\mathrm{OR}}$ & & $\mathrm{I}^{2}(\%)$ & $\mathrm{P}_{\mathrm{H}}$ \\
\hline \multirow{6}{*}{$\begin{array}{c}\text { All relevant } \\
\text { articles }\end{array}$} & Total & A vs $\mathrm{T}$ & 0.693 & $(0.515-0.933)$ & 0.016 & $\mathrm{~F}$ & 22.7 & 0.256 \\
\hline & & AA vs TA+TT & 1.482 & $(1.085-2.023)$ & 0.022 & $\mathrm{~F}$ & 0.00 & 0.435 \\
\hline & & AA vs TA & 1.491 & $(1.085-2.048)$ & 0.014 & $\mathrm{~F}$ & 0.00 & 0.467 \\
\hline & Caucasian & A vs T & 0.803 & $(0.577-1.117)$ & 0.193 & $\mathrm{~F}$ & 0.00 & 0.515 \\
\hline & & $\mathrm{AA}$ vs TA+TT & 1.297 & $(0.917-1.835)$ & 0.141 & $\mathrm{~F}$ & 0.00 & 0.676 \\
\hline & & AA vs TA & 1.333 & $(0.935-1.899)$ & 0.112 & $\mathrm{~F}$ & 0.00 & 0.596 \\
\hline \multirow{6}{*}{$\begin{array}{l}\text { Excluded } \\
\text { for HWE }\end{array}$} & Total & A vs $\mathrm{T}$ & 0.790 & $(0.568-1.098)$ & 0.160 & $\mathrm{~F}$ & 0.00 & 0.451 \\
\hline & & $\mathrm{AA}$ vs $\mathrm{TA}+\mathrm{TT}$ & 1.344 & $(0.952-1.897)$ & 0.093 & $\mathrm{~F}$ & 0.00 & 0.605 \\
\hline & & AA vs TA & 0.736 & $(0.519-1.043)$ & 0.085 & $\mathrm{~F}$ & 0.00 & 0.687 \\
\hline & Caucasian & A vs $\mathrm{T}$ & 0.842 & $(0.590-1.202)$ & 0.344 & $\mathrm{~F}$ & 0.00 & 0.425 \\
\hline & & AA vs TA+TT & 1.284 & $(0.952-1.897)$ & 0.189 & $\mathrm{~F}$ & 0.00 & 0.513 \\
\hline & & AA vs TA & 0.752 & $(0.515-1.097)$ & 0.350 & $\mathrm{~F}$ & 0.00 & 0.538 \\
\hline
\end{tabular}

Table 2 Pooled Measures on the Relations of TFAM gene rs1937 Polymorphisms to Alzheimer's disease risk.

CI, confidence interval; DHWE,deviated from HWE in controls; FEM, fixed effect model;

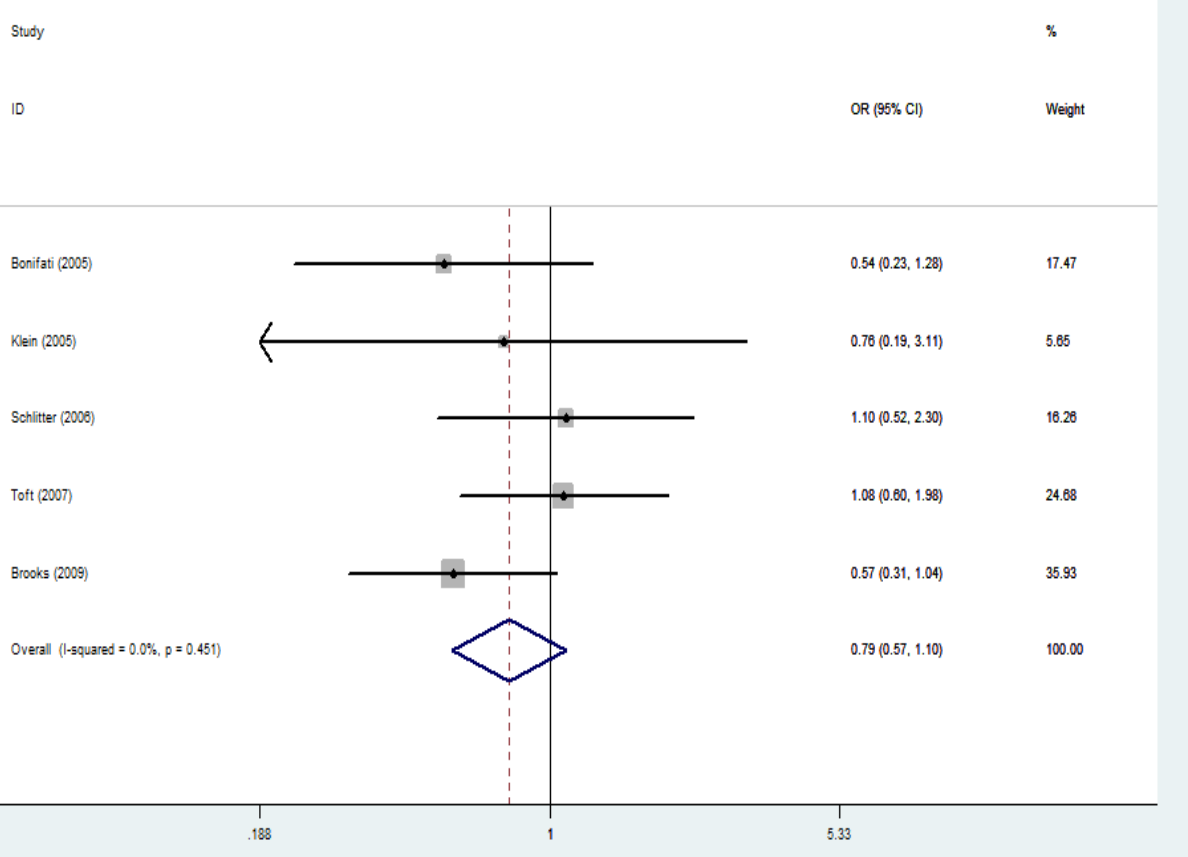

Fig 2 Forest plot for the relationship of PINK1 Q115L polymorphism and the risk of PD under the allele model after exclusion of articles deviating from HWE in controls. 


\section{Sensitivity analysis and publication bias}

A sensitivity analysis was additionally conducted in which every study was removed in turn. The results showed no significant alterations in pooled OR and 95\% CI values in all genetic models, which indicated high stability of this meta-analysis. Funnel plot and Begg's test was utilized to assessed the publication bias of the inclued studies. No publication bias was observed in all genetic models. The shape of funnel plot for meta-analysis of PINK1 Q115L in the included studies under all genetic models seemed symmetrical. Begg's test indicated no statistical evidence of publication bias among studies (data not shown), suggesting no obvious publication bias.

\section{Influence analysis}

No individual study was found to have excessive influence on the pooled effect in the three above-mentioned inherited models before and after excluding the article that deviated from HWE in controls .

\section{Discussion}

The PINK1 gene encodes a putative protein kinase which is targeted to mitochondria and shows a serine-threonine kinase domain with homology to kinases of the $\mathrm{Ca} 2+/$ calmodu lin family[8]. It appears to exert protective effects against cellular stress within mitochondria(1). The mechanism of PINK1's suggested ability to prevent cell death is by the inhibition of release of cytochrome $\mathrm{C}$ from mitochondria and maintenance of the mitochondrial membrane potential[8, 22-23]. Mitochondrial dysfunction is considerd to play an integral role in the pathogenesis of $\mathrm{PD}[24]$.

In recent years, several studies had been performed to evaluate the relationship between the Q115L polymorphism in the PINK1 gene and PD. However, the results in the publications remain controversial. Because individual studies have relatively small numbers of participants with underpower to detect the effect, a meta-analysis may be the appropriate approach to obtain a more definitive conclusion regarding the role of the PINK1 gene Q115L polymorphism on risk of PD. For all we know, this is the first meta-analysis carried out to study the relationship between the
PINK1 Q115L genetic polymorphism and PD.

In the present meta-analysis, there was not exist some relationships between the PINK1 Q115L polymorphism and PD risk after exclusion of articles deviating from HWE in controls. In a further subgroup analysis restricted to the ethnic of PD patients, no significant increased risk was found in patients in Caucasian. When interpreting the results, several limitations which may have affected the objectivity of the conclusions should be taken into account.

Firstly, the accuracy of clinical diagnostic does not reach $80 \%$, caused by varying levels of expertise of researchers. Some PD patients recruited for the studies may be misclassified as cases of Parkinson-plus syndromes or Essential Tremor. This misdiagnose may alter the results of studies. secondly, though many factors were reported to be involved in PD, we did not take all these factors into our analysis, for example, environmental and genetic factors. Thirdly, a language bias may have existed because this meta-analysis only contained English languages articles. Nevertheless, some studies in coincidence with the inclusion criteria in other languages published in specific journals could not be identified and included in this meta-analysis.

In summary, our meta-analysis provided some evidence that the PINK1 Q115L polymorphism might not has associations with PD. In order to get a better understanding about the mechanisms of PD, further studies with larger samples across more countries are required. It will be necessary to take genetic factors, ethnicity, gender, and environmental risk factors into consideration.

\section{Acknowledgements}

This work was supported by grants from the 973 program (2011CB504102), the National Foundation of Natural Science of China (31471114), Program for New Century Excellent Talents in University and Taishan Scholar Program.

\section{References:}

1. Van Den Eeden, S.K., et al., Incidence of Parkinson's disease: variation by age, gender, and race/ethnicity. Am J Epidemiol, 
2003. 157(11): p. 1015-22.

2. Olanow, C.W., M.B. Stern, and K. Sethi, The scientific and clinical basis for the treatment of Parkinson disease (2009). Neurology, 2009. 72(21 Suppl 4): p. S1-136.

3. Tolleson, C.M. and J.Y. Fang, Advances in the mechanisms of Parkinson's disease. Discov Med. 15(80): p. 61-6.

4. Varcin, M., et al., Oxidative stress in genetic mouse models of Parkinson's disease. Oxid Med Cell Longev. 2012: p. 624925.

5. Blesa, J., et al., Classic and new animal models of Parkinson's disease. J Biomed Biotechnol. 2012: p. 845618.

6. Gwinn-Hardy, K., Genetics of parkinsonism. Mov Disord, 2002. 17(4): p. 645-56

7. Pankratz, N. and T. Foroud, Genetics of Parkinson disease. Genet Med, 2007. 9(12): p. 801-11.

8. Valente, E.M., et al., Hereditary early-onset Parkinson's disease caused by mutations in PINK1. Science, 2004. 304(5674): p. 1158-60.

9. Sim, C.H., et al., C-terminal truncation and Parkinson's disease-associated mutations down-regulate the protein serine/threonine kinase activity of PTEN-induced kinase-1. Hum Mol Genet, 2006. 15(21): p. 3251-62.

10. Valente, E.M., et al., PINK1 mutations are associated with sporadic early-onset parkinsonism. Ann Neurol, 2004. 56(3): p. $336-41$.

11. Bonifati, V., et al., Early-onset parkinsonism associated with PINK1 mutations: frequency, genotypes, and phenotypes. Neurology, 2005. 65(1): p. 87-95.

12. Healy, D.G., et al., PINK1 (PARK6) associated Parkinson disease in Ireland. Neurology, 2004. 63(8): p. 1486-8.

13. Brooks, J., et al., Parkin and PINK1 mutations in early-onset Parkinson's disease: comprehensive screening in publicly available cases and control. J Med Genet, 2009. 46(6): p. $375-81$.

14. Ishihara-Paul, L., et al., PINK1 mutations and parkinsonism. Neurology, 2008. 71(12): p. 896-902.

15. Klein, C., et al., PINK1, Parkin, and DJ-1 mutations in Italian patients with early-onset parkinsonism. Eur J Hum Genet, 2005. 13(9): p. 1086-93.

16. Schlitter, A.M., et al., Exclusion of PINK1 as candidate gene for the late-onset form of Parkinson's disease in two European populations. J Negat Results Biomed, 2005. 4: p. 10.

17. Toft, M., et al., PINK1 mutation heterozygosity and the risk of Parkinson's disease. J Neurol Neurosurg Psychiatry, 2007. 78(1): p. 82-4.

18. Higgins, J.P., et al., Measuring inconsistency in meta-analyses. BMJ, 2003. 327(7414): p. 557-60.

19. Peters, J.L., et al., Comparison of two methods to detect publication bias in meta-analysis. JAMA, 2006. 295(6): p. 676-80.

20. Huxley, N., et al., A systematic review and economic evaluation of intraoperative tests [RD-100i one-step nucleic acid amplification (OSNA) system and Metasin test] for detecting sentinel lymph node metastases in breast cancer. Health Technol Assess, 2015. 19(2): p. v-xxv, 1-215.

21. Patsopoulos, N.A., E. Evangelou, and J.P. Ioannidis, Sensitivity of between-study heterogeneity in meta-analysis: proposed metrics and empirical evaluation. Int J Epidemiol, 2008. 37(5): p. 1148-57.

22. Petit, A., et al., Wild-type PINK1 prevents basal and induced neuronal apoptosis, a protective effect abrogated by Parkinson disease-related mutations. J Biol Chem, 2005. 280(40): p. 34025-32.

23. Duchen, M.R., A. Surin, and J. Jacobson, Imaging mitochondrial function in intact cells. Methods Enzymol, 2003. 361: p. 353-89.

24. Onyango, I.G., Mitochondrial dysfunction and oxidative stress in Parkinson's disease. Neurochem Res, 2008. 33(3): p. 589-97. 